\title{
Long Axis
}

National Cancer Institute

\section{Source}

National Cancer Institute. Long Axis. NCI Thesaurus. Code C94857.

Line through and in the direction of an object's characteristic feature that is longer than any other feature. 